\title{
The Role of Cognitive Modeling in Enhancing Dynamic Decisions
}

\author{
Cleotilde Gonzalez (coty@cmu.edu) \\ Social and Decision Sciences Department \\ Carnegie Mellon University \\ 5000 Forbes Ave. Pittsburgh, PA 15213-3890 USA
}

Dynamic Decision Making is characterized by multiple and interdependent decisions, autonomous environments, and real-time evaluation and action (Brehmer, 1990). There seems to be increasing agreement on how we make decisions in this type of situations. Decision makers in dynamic environments recognize typical situations and typical responses and use their knowledge to adapt their strategies "on the fly" (Payne, Bettman, and Johnson, 1993; Klein, 1998).

Many cognitive models have been developed in the past decade to investigate different aspects of cognition in these environments: attention and multitasking (Lebiere, Anderson and Bothell, 2001; Altmann and Gray, 2000); judgment and choice in decision making (Gonzalez, Lerch and Lebiere, submitted); and skill acquisition in dynamic situations (Wallach and Lebiere, 2000; Schoppek, Holt, Diez and Boehm-Davis, 2001). These efforts are commendable for focusing on complex, real world tasks. However, the role of cognitive modeling in enhancing decision-making in these situations has been very static and limited.

Traditionally, cognitive models are developed within the context of a task. A synthetic version of the task exists in the form of a computer program, and a cognitive model is developed to interact with such a tool. On the other hand, the synthetic task environment supports the collection of behavioral data and the development of the cognitive model. Theories expand and conclusions are reached about human cognition based on the comparisons between human and model data. I believe that cognitive modeling may and should take a more dynamic role in enhancing decisionmaking. Creating cognitive models to perceive a situation, combine goals and beliefs, choose a course of action, and react over the environment, is a challenge that we should overcome.

In my current research, I intend to enhance existent cognitive models to take an active, real-time role in decision making and learning. An example of this effort is the cognitive model of instance-based decision-making, developed in the Water purification Plant (WPP) task (Gonzalez, Lerch and Lebiere, submitted). WPP is a resource allocation, scheduling task, isomorph of a real world task in an organization with large-scale logistical operations (the United States Postal Service: USPS) (Lerch, Ballou, and Harter, 1997). WPP is highly dynamic because of exogenous events occurring without the decision maker's control and it is complex because of the number of options and alternatives to consider at any given time. All these elements interact with the decision maker's actions in a realtime environment, and events occur as the simulation clock is running. Based on an Instance-Based Decision Making framework, I have developed a cognitive model (built on ACT-R) to interact with the WPP task. At present, the model accurately simulates the variability of choice in this dynamic situation and allows strategy adaptation and flexibility. But, the model works off-line, not directly incorporated in monitoring the status of a real decision maker in real-time.

I intend to use this cognitive model to help track and adapt the conditions of WPP in real-time, according to the cognitive necessities of a human decision maker interacting with the task. By tracking each of the actions taken by a human decision maker and monitoring the status of the task environment, the cognitive model would be able to predict the possible contents of working memory and the state of knowledge in the form of instances (See figure). Based on the predicted cognitive status of human decision makers, the cognitive model may give advice to the decision maker in the form of:

1. attracting attention to the parts of the screen that have not been acted on and require awareness according to the environmental status

2. Presenting information in multiple modalities to spread the load to multiple channels

3. Perform urgent actions that the decision maker would not have time or resources to react to

Although simple and limited, I believe that, this effort may demonstrate an active and dynamic role of cognitive modeling in complex situations.

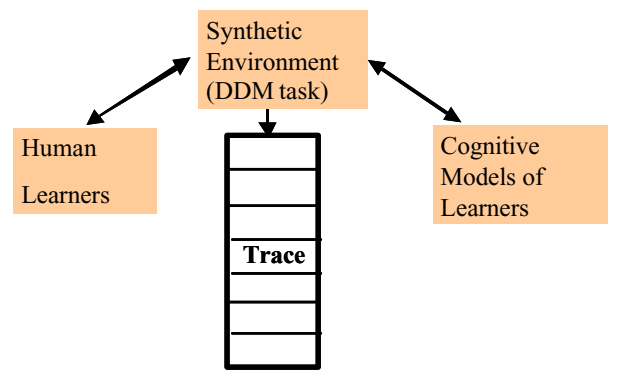

\section{References}

Due to space limitations, I have not included the references. Please request this list to: coty@cmu.edu 